\title{
Correction: Dynamic Endothelial Cell Rearrangements Drive Developmental Vessel Regression
}

Claudio A. Franco, Martin L. Jones, Miguel O. Bernabeu, Ilse Geudens, Thomas Mathivet, Andre Rosa, Felicia M. Lopes, Aida P. Lima, Anan Ragab, Russell T. Collins, Li-Kun Phng, Peter V. Coveney, Holger Gerhardt

An affiliation listed for the last author is incorrect. Holger Gerhardt is affiliated with the Vesalius Research Center (\#6) rather than the University of Edinburgh (\#5). The correct affiliations are as follows:

1. Vascular Biology Laboratory, London Research Institute-Cancer Research UK, Lincoln's Inn Fields Laboratories, London, United Kingdom,

6. Vascular Patterning Laboratory, Vesalius Research Center, KU Leuven, Department of Oncology, VIB3, Leuven, Belgium

Current address: Max-Delbrück Center for Molecular Medicine, Berlin, Germany

\section{Reference}

1. Franco CA, Jones ML, Bernabeu MO, Geudens I, Mathivet T, Rosa A, et al. (2015) Dynamic Endothelial Cell Rearrangements Drive Developmental Vessel Regression. PLoS Biol 13(4): e1002125. doi: 10. 1371/journal.pbio.1002125 PMID: 25884288

G OPENACCESS

Citation: Franco CA, Jones ML, Bernabeu MO, Geudens I, Mathivet T, Rosa A, et al. (2015) Correction: Dynamic Endothelial Cell Rearrangements Drive Developmental Vessel Regression. PLoS Biol 13(5): e1002163. doi:10.1371/ journal.pbio.1002163

Published: May 14, 2015

Copyright: ๑ 2015 Franco et al. This is an open access article distributed under the terms of the Creative Commons Attribution License, which permits unrestricted use, distribution, and reproduction in any medium, provided the original author and source are credited. 